\title{
Phenomenal Consciousness and the Sensorimotor Approach. A Critical Account
}

\author{
Alessandro Dell'Anna ${ }^{1}$, Alfredo Paternoster ${ }^{2}$ \\ ${ }^{1}$ Department of Philosophy, University of Genova, Genova, Italy \\ ${ }^{2}$ Department of Arts Philosophy, University of Bergamo, Bergamo, Italy \\ Email: a_dellanna@hotmail.com, alfredo.paternoster@unibg.it
}

Received July $17^{\text {th }}$, 2013; revised August $17^{\text {th }}$, 2013; accepted August $25^{\text {th }}, 2013$

\begin{abstract}
Copyright @ 2013 Alessandro Dell'Anna, Alfredo Paternoster. This is an open access article distributed under the Creative Commons Attribution License, which permits unrestricted use, distribution, and reproduction in any medium, provided the original work is properly cited.
\end{abstract}

\begin{abstract}
The paper discusses some recent suggestions offered by the so-called sensorimotor (or enactivist) theorists as to the problem of the explanatory gap, that is, the alleged impossibility of accounting for phenomenal consciousness in any scientific theory. We argue in the paper that, although some enactivist theorists' suggestions appear fresh and eye-opening, the claim that the explanatory gap is (dis)solved is much overstated.
\end{abstract}

Keywords: Sensorimotor Approach; Enaction; Phenomenal Consciousness; Externalism; Explanatory Gap

\section{Introduction}

In this paper we shall discuss whether the sensorimotor approach to perceptual experience, in its different versions (O’Regan \& Noë, 2001; Noë, 2004; Thompson, Palacios, \& Varela, 1992; see also Hurley, 1998) has the theoretical resources to address the problem of consciousness, and in particular the so-called explanatory gap, as some of its proponents argued (see e.g. O’Regan, Myin, \& Noë, 2005; Thompson, 2005; Thompson \& Varela, 2001). We shall argue that the sensorimotor approach provides some evidence for the role of external (non-cranial and extra-bodily) factors in the "production" of conscious experience, thereby restating the issue to some extent, but it is not able to definitely remove, or deflate, the so-called explanatory gap.

In the first section we shortly introduce the sensorimotor account, focusing on the aspects relevant to the issue of consciousness. The second section is devoted to a critical discussion of the two main arguments offered by sensorimotor theorists aiming to show that the explanatory gap can be removed or explained away. In the third section we qualify the kind of externalist strategy pursued by sensorimotor theorists to deal with the problem of conscious experience and we assess to what extent it is successful. In the fourth section we discuss a different strategy for dealing with the problem of consciousness in the sensorimotor framework. In the last section we draw some conclusions.

\section{The Sensorimotor Account of Perceptual Experience}

Although the expression "sensorimotor account" was introduced by O’Regan and Noë (2001) and, narrowly interpreted, it denotes their specific view on perception, in a broader sense we can regard the sensorimotor approach as a family of theories agreeing on a critical attitude as regards computational theories of perception (and to classical computationalism in general). Sensorimotor theories include the approach of enactive vision (see e.g. Thompson, Palacios e Varela, 1992; Noë, 2004; Thompson, 2007), the research program of animate vision (Ballard, 1991, 1996) and the behavior-based approach (or situated robotics: Brooks, 1991, 1999). Dynamical approaches to cognitive science (for instance, Thelen, \& Smith, 1994; Kelso, 1995) can also be traced back, in certain aspects, to the sensorimotor paradigm.

In this paper we focus on those versions of the theory that are most relevant to the issue of consciousness, that is, the sensorimotor approach in the narrow sense of the term or the so-called enactive vision. The core of this view can be characterized by the conjunction of the two following theses:

1) Perception is the activity of exploring the environment, an activity which is carried out by exploiting a systematic interdependency of sensorial information and motor behavior. Much in the spirit of ecological optics (Gibson, 1979), every kind of action structures the optic array (in the case of vision) in a peculiar lawful way.

2) Perception is constituted by, rather than merely depending on, the possession of sensorimotor knowledge. This knowledge consists in the ability to perform movements appropriate to a successful "navigation" of the environment, including in this latter notion the pursuit of basic ecological goals such as grasping an object. Sensorimotor knowledge is tacit and determined by bodily structure.

Taken together, these two theses can be summarized by saying that perception is a kind of activity that is performed thanks to the possession of a certain kind of physical competence or ability, a kind of bodily know-how. What we perceive is determined by what we do, and what we do is part of the perceptual process.

The leading intuition in the sensorimotor view is that there is a sort of loop in our sensorimotor relation to the world: on the 
one hand, changes in the stimulation modify our experiencethings appear different; on the other hand, agents' movements change the stimulation (and experience as well). Indeed changes in the stimulation typically require agents to move, for example, in order to keep a moving object in the focus of attention. It is for this reason that the content of sensorimotor ability consists essentially of the knowledge of how one has to move in order to track changes. We could say that an important component of perception is anticipatory, since agents are competent in the sensorimotor domain to the extent they know how stimulation will change following movement. Changes in stimulation according to variations of the body (and vice-versa) are called "sensorimotor dependencies", or (less perspicuously) "sensorimotor contingencies".

An important implication of such a view is that perception is not a brain process, but, rather, a competent activity performed by the whole animal. This is the main point of departure from (classical) computationalism, which tended to identify the perceptual system with a part of the nervous system (though described at an algorithmic level).

Now, whatever may be the other merits and shortcomings of the computational approach in the domain of perception, it must be recognized that computationalism has many difficulties in dealing with consciousness. The basic reason for this is that computational functionalism has deliberately put aside the conscious aspect of mental processes, in at least three senses. First of all, whether a process is conscious or not is definitely not relevant for a computational theory. In fact, computational theories are typically descriptions of subpersonal processes. Perhaps some pieces of these processes emerge at the level of consciousness, but this is not necessary and does not make a difference for the functional-computational nature of the process. Second, some authors have explicitly endorsed the assumption that the best strategy for addressing the study of mind is to separate intentionality (or "content”) from consciousness (cf., for instance, Dennett, 1969; Fodor, 1980). As a matter of fact, this separation has for many years involved addressing just intentionality, leaving consciousness apart. Third, paradigmatic phenomenal states, such as pain, can hardly be accounted for in purely functional terms; so, computational theories, qua functional, are not able to deal with phenomenal states, or with the phenomenal aspect of mental states. In a nutshell, in the computational account consciousness turns out to be a bothersome leftover.

Of course there is a ruthless way to reply to these considerations, consisting of the rejection of the premise according to which there is something like a phenomenal quality to account for (Dennett, 1991, 2005). Although we believe that Dennett is right on some important points (cf. infra), it seems to us that the concept of phenomenal consciousness cannot be simply ruled out in the way it is by Dennett.

Are sensorimotor approaches in a better position to deal with the problems of consciousness? We shall consider, to begin with, some arguments put forward by the most representative supporters of the sensorimotor approach (Hurley, 1998; Hurley \& Noë, 2003; Noë, 2004; Noë \& Thompson, 2004; O’Regan, Myin, \& Noë, 2005; Myin \& O’Regan, 2008)—what Clark (2008) calls the "strong sensorimotor approach". These arguments aim to show that the sensorimotor approach is able to close, or at least to deflate, the "explanatory gap", that is, the alleged impossibility of accounting for phenomenal consciousness in terms of whatever scientific (psychological or neuron- logical) explanation (Levine, 1983; Chalmers, 1996).

\section{Two Arguments for Removing (or Explaining Away) the Explanatory Gap}

The core intuition of enactivists as to the problem of the explanatory gap can easily be stated in a phrase: (phenomenal) consciousness does not come from the brain, so there is no explanatory gap at all, since the alleged gap is regarded to be between the brain and the person. The idea is that if one gives up the assumption that consciousness supervenes only onto the brain, then there is no gap any longer: the question how could such and such neural state give rise to such and such feeling is misleading, because, although the neural state has a causal role in producing the feeling, it does not exhaust the base of supervenience. Brains are at most a causal (but not constitutive) factor of consciousness, since, "rather than directly producing phenomenal experience, neural activity is involved in phenomenal experience because of what it allows organisms to do" (O'Regan, Myin, \& Noë, 2005: p. 371), and "the perceived quality of sensory stimulation is determined by the particular way subjects interact with their surroundings rather than by the specific character of any intervening brain processes or representations" (Myin \& O’Regan, 2008: p. 192).

Why should we believe that the supervenience base of "the perceived quality" includes (to say the least) bodily know-how and external factors? It seems to us that two main arguments can be traced in the discussion of sensorimotor theorists: the argument from the virtual nature of experience (briefly, the argument from virtual experience), and the argument from sensory substitution (which we call, for brevity, the "TVSS argument”, since the discussion usually concerns Paul Bach-y-Rita (1972) Tactile Vision Sensory Substitution system). Let us discuss each of them, one at a time.

\section{The Argument from Virtual Experience}

According to sensorimotor theorists experience is virtual in the sense that experience is in part constituted by what one could do, rather than being only constituted by actual feelings. For example, when your fingers are in contact with some points of the surface of an object, you have the sensation of perceiving the object as a whole, i.e., you have the feeling of grasping the entire object, although you are actually in contact only with some parts of its surface. This is an instance of the general, well-known phenomenon of amodal completion (see e.g. Kanizsa, 1979). On the sensorimotor view, amodal completion can be explained by our knowing what we could do with the object by exploring it, that is, by our sensorimotor ability (O’Regan, Myin, \& Noë, 2005). The same explanation is taken to account also for a different kind of "completion", the fact that we do not experience any "visual gap" although there is the so-called blind spot ("macula cieca") in the retina: we have visual experiences of certain features that are actually not present in the proximal stimulation, since they would fall in the region, devoid of photoreceptors, where the optic nerve departs.

Therefore, it could be argued that the experience of an object depends on the sensorimotor knowledge, rather than being directly provided by some neural activity. Far from being constituted by what actually happens, experience is in part constituted by what one could do, something that, as virtual, cannot have an actual cerebral realization. As Alva Noë put it, "my sense of the perceptual presence of items at the periphery of my visual 
field... is not a sense that I actually see these features, but that I have access to them, due to the fact that my relation to them is mediated by patterns of sensorimotor contingency" (2004: p. 216 , our italics). One can talk about a perceptual virtual presence since, even if one cannot really see a virtual item, one has rather some (normally satisfied) expectations: virtual presence is a dispositional property which, however, is usually made actual some instants later.

The argument of virtual experience is strengthened by the remark that perceptual experience has two phenomenal features. First, sensorial information suddenly changes as soon as one performs the slightest movement. This property, called by O’Regan, Myin \& Noë (2005) “corporality”, or "bodiliness”, would explain the "intimate" quality of perceptual experience: "because sensory information is so exquisitely sensitive to body motions, it is almost as though it were part of you" (ibid., p. 374). The second feature, called "alerting capacity" or, in other essays, “grabbiness” (see e.g. Myin \& O’Regan, 2008), consists of the very fast focalization of attention when there are sudden changes in the stimulation. In the visual case, for instance, a change in the visual field triggers a movement of the eye so that the fovea turns out to be lined up to the location of the change. Thus, a visual variation is immediately recognized and analyzed. Grabbiness makes all parts of the visual field very vivid and present (again, intimately related to us). In this way, certain qualitative aspects of experience-those aspects that make the experience mine (cf. e.g., Metzinger 2003)-are explained in terms of objective features of the way our perceptual systems work.

It is not very clear whether the notions of corporality and grabbiness are intended to be applied to the "real" experience rather than to the virtual one. They are perhaps better to be considered as further evidence for the thesis that the supervenience basis of (phenomenal) consciousness is crucially constituted by sensorimotor competence, i.e., by a bodily know-how. Be that as it may, the phenomenon of virtual presence would show that experience does not supervene on neural facts, since, for instance, the hidden face of a perceived object is not actually available to photoreceptors nor, then, to the brain. Of course there are some parts of the experiential content which are "actual", but the point is that the possibility of being present to consciousness despite not being available to receptors is a sufficient condition to show that (in general) experience need not possess a cerebral basis. As we said above, the problem of the explanatory gap would vanish insofar as sensation is not regarded as being supervenient on neurophysiological patterns. It is worth to point out that this argument concerns specifically perceptual experience, as opposed to the experience of having a thought, or to the experience of pain. Thus, even if the argument were sound, it would be limited in scope.

Now, it could be replied, first of all, that the most plausible causal source of the fact that something is present to consciousness, despite not being really (“objectively”) present in the portion of environment available to receptors is after all the brain. The hypothesis that the alleged virtual part of experience is produced by the brain not only makes sense, but seems (prima facie) to be the most likely one, as is particularly apparent in the case of the blind spot (see, e.g., Churchland \& Ramachandran, 1996).

It must be conceded, however, that the case of blind spot is not exactly analogous to the case of amodal completion (the notion of virtual seems to fit better the latter rather than the former), and that there is at least some plausibility in the enactivist account of amodal completion. For, as we said above, there is a clear sense in which a hidden part is not actually available to the brain despite of being phenomenally "available" (to the whole person). As far as the issue of the nature of the supervenience basis is concerned, it is not very clear who has the burden of proof.

However, even if one grants this point to sensorimotor theorists, the argument from virtual experience seems to face another serious difficulty. Clark (2009) discharges the argument from virtual experience for-he claims-it faces the following dilemma: either experience is only in part virtual, or experience is entirely virtual. If the former, then the explanatory gap is still there because we need an explanation of how the real (= non-virtual) experience is produced. If the latter, the problem is that we lack a clear justification of the claim that experience is always virtual, which appears (at least prima facie) hardly believable. Noë (2006) claimed that experience is virtual "all the way in” (p. 421), but, as Clark correctly points out, this is absolutely obscure. Even if what is meant by Noë is that "what fixes any experience is not a snapshot moment of neural activity but a process extended in time (...) then all that seems to matter is that the neural activity evolves over time in suchand-such way” (Clark, 2009: pp. 974-975).

In sum, what the argument from virtual experience at most shows is that there are non-cranial factors which are relevant to explain the phenomenon of virtual experience, but this does not undermine at all the thesis that non-virtual experience is determined by the cerebral machinery.

\section{The TVSS Argument}

Sensorimotor theorists take the experiments on the so-called sensory substitution systems, such as the famous, pioneering TVSS (Bach-y-Rita, 1972), as further evidence for the thesis that consciousness is not (only) produced by the brain. In the TVSS blind subjects are able to feel visual-like experiences through a tactile perception system arranged on their shoulder. For instance, a typical visual feature echoed in their experience is spatial localization: after training, subject feel the objects located in the external space, rather than in their skin; and they entertain typical visual feelings such as parallax, zoom, perspective. As Hurley \& Noë (2003) point out, in structural respects TVSS-perception after adaptation is more like vision than it is like touch. In fact, both in ordinary vision and in TVSS-vision, we make perceptual contact with objects arrayed out before us at a distance in space: unlike touch, there is no immediate physical contact with perceptual objects.

Why are sensory substitution systems taken to provide evidence for the failure of supervenience of experiential content onto the brain? Because they would seem to show that the quality of experience does not depend on the activity of some specific brain areas, but, rather, on the sensory system organization. According to O’Regan, Myin \& Noë (2008), for instance, neural activation is just a way to code information, and it is hard to see why neural activation in (brain-area) A rather than in B should make a difference in perceptual quality; intermodal differences in experiential quality are better explained by differences in sensorimotor dependencies. Thus, the prediction is that, if we replace one sensory system (say, a visual system) with another (say, a tactile one) and keep the sensorimotor dependencies of the former, we shall obtain the quality of the former. 
The crucial point is that TVSS is a "quasi-visual" modality since it shares with vision a common sensorimotor structure. "Changes in qualitative expression are to be explained not just in terms of the properties of sensory inputs and of the brain region that receives them, but in terms of dynamic patterns of interdependence between sensory stimulation and embodied activity. What drives changes in qualitative expression of a given area of cortex (...) is not simply a remapping from the sources of input, whether internal or external, to that area of cortex, but rather higher-order changes, in relations between mappings from various different sources of input to different areas of cortex and from cortex back out to effects on those sources of input, which are in turn fed back to various areas of cortex" (Hurley \& Noë, 2003: p. 146).

However, the quasi-visual nature of the TVSS experience can seriously be called into question: if it is true that some visual features are actually reproduced, other salient visual features, such as color, are not, and, on the whole, the quality of the TVSS-experience is still quite different from "normal" visual experience-in a word, TVSS subjects do not really see, as Bach-y-Rita himself was quite ready to concede.

Of course, supporters of the sensorimotor paradigm are well aware of this difficulty. They reply by saying that the partial inadequacy of the TVSS as a visual tool depends, at least in part, on the fact that the system is not sophisticated enough. Moreover, they reject the idea that visual experience is a matter of “all or nothing” (O’Regan \& Noë, 2001: p. 958): it is true that color and stereo vision are absent, and resolution is extremely poor. But, just as color blind, stereo blind, one-eyed or low-sighted people can be said to "see," people using the TVSS should also be said to see. Seeing with the skin probably involves laws that are not exactly the same as seeing with the eyes, just as seeing colors in the dark is not quite the same as in the light ${ }^{1}$.

Yet, quite independently of the way the experiential quality of the TVSS is assessed, it seems to us that the role played by the brain in the production of experience is underestimated: even if one acknowledges that neural activation is just a way of coding information, still the fact that certain areas receive projections from, say, A-receptors rather than B-receptors, does make a difference, and this is an internal difference. There is no compelling evidence from the TVSS for the failure of supervenience of experiential content onto the brain ${ }^{2}$. Today we know that there are many projections from cortical areas back to the periphery of the nervous system, so it comes not as a surprise that knowledge flowing from the center permeates what the senses detect. Moreover this would corroborate the anticipatory character of sensorimotor knowledge, that Noë himself is emphasizing, and is coherent with an important remark O’Regan and Noë (2001: p. 944) make in order to clarify the sensorimotor contribution to consciousness: "For a creature (or a machine for that matter) to possess visual awareness, what is required is that, in addition to exercising the mastery of the relevant sensorimotor contingencies, it must make use of this exercise for the purpose of thought and planning" (our emphasis). That is, the simple exploitation of a sensorimotor contin-

\footnotetext{
${ }^{1}$ Indeed, it could be argued that the TVSS is a new kind of sensory system, neither visual nor tactile.

${ }^{2}$ The experiments on rubber arms (see e.g. Botvinick \& Cohen, 1998; Ramachandran \& Blakeslee, 1998) are also regarded as (perhaps equally controversial) cases against the brain supervenience of phenomenal consciousness.
}

gency remains a sub-personal process, whereas its merging with thought processes makes it poised for consciousness. We assume that thought processes, qua higher-order mental processes, are implemented in higher areas of the brain, providing the feedback that a virtual presence, as Noë would have it, might require.

Clark (2009) has an even stronger reply to the argument from TVSS. He claims that the argument from TVSS falls into the fallacy of mistaking a causal property for a constitutive property. As he put it, the argument from TVSS “depends on taking evidence for the role of whole sensorimotor loops in training and tuning the neural systems that support conscious perception for evidence of the ongoing role of such loops (...) in conscious perception itself" In other words, consciousness depends on neural modifications; sensorimotor loops are the causal sources of these modifications, but the "real work" is made by neural circuits: if neural circuits were not affected by sensorimotor loops, the quasi-visual consciousness would hardly be produced.

The causal-for-constitutive fallacy is familiar in the debate on (various kinds of) externalism in philosophy of mind. Indeed, the typical argument for externalism on intentional content rests on the intuition that a difference in the environment can make a mental difference despite of all internal factors being equal ${ }^{3}$. Internalists, however, can reply to this that a difference in environment makes a mental difference only provided that the environmental difference is taken into account by sensory systems. For instance, if a change in a distal condition does not yield a corresponding change in the proximal stimulus-in the retinal image-intentional content will not change (see, e.g., McGinn 1989). Of course, an environmental change normally determines a proximal change, but this is not the case in the counterfactual hypothesis set out in thought-experiments. Indeed, in order to test our intuitions on how intentional content should be individuated, modal considerations are crucial. When, as usual, there is a causal chain of events which determine, in the end, a certain kind of mental event, all the links of the chain are causally relevant, but the distal cause-the environmental condition-is "screened off" by the proximal condition. That's why the distal condition has a causal but not constitutive role, as the counterfactual scenario makes explicit.

Yet, there is a move available to externalists, and they actually made it: they can argue that our ways to ascribe mental contents imply that the distal/causal element is part of the intentional content. That would make the distal causal factor content-constitutive. Is this reasonable? And, supposing it is so, is this move available even in the case of phenomenal content?

\section{Sensorimotor Accounts and Varieties of Externalism}

We saw two arguments aiming to show that the sensorimotor approach can remove or dissolve the explanatory gap, and we rose some doubts on the plausibility of these arguments. Maybe other arguments can be reconstructed from the examples discussed by enactivist scholars (see, e.g., what Clark 2009 calls the "argument from the variable neural correlate"), but, in essence, all sensorimotor considerations and examples are instances of one and the same strategy: arguing for some kind of

${ }^{3}$ Here we are referring to perceptual content. We leave apart belief content. Perspectives of externalism are arguably worse in the case of belief content. 
externalism as to phenomenal facts.

Externalism on phenomenal facts is regarded as a sufficient condition for removing the explanatory gap ${ }^{4}$. Therefore, two questions are in order here: 1) do the arguments from virtual experience and from TVSS provide evidence for endorsing externalism of phenomenal consciousness? 2) Is externalism of phenomenal content indeed sufficient for closing the gap?

As we are going to argue, our answers are "maybe" (or, "it depends") to the first question, and "not at all" to the second one. Even if one wishes to concede that some version of externalism as to phenomenal facts is true, still it is hard to understand why externalism of phenomenal content should be sufficient for dissolving the explanatory gap, since to say that the brain does not exhaust the supervenience base does not amount to say, of course, that brain is not involved at all.

We could describe the situation as follows. According to sensorimotor theorists, the content of perception is determined by sensorimotor ability (or competence), and sensorimotor ability involves crucially external factors, thereby justifying externalism as to phenomenal content. However, there are two problems. First, the enactivist strategy faces the following dilemma: either sensorimotor competence is a (genuine kind of) knowledge represented in the brain, or sensorimotor competence is distributed (rather than represented) as is claimed, for instance, in the extended mind model (Clark \& Chalmers, 1998). If the former, of course, externalism fails and the explanatory gap is still there (this corresponds to the causal/constitutive fallacy); therefore, we can take for granted that sensorimotor theorists endorse the second horn of the dilemma. And here we face the second problem: enactivists have to show that the external "components" of sensorimotor ability determine also the phenomenal aspect of perceptual content.

As we saw in the previous section, however, the inference from the cases discussed by sensorimotor theorists to externalism as to phenomenal content (please take hereafter the expression "phenomenal content" as short for "phenomenal component of mental content") cannot easily be drawn. Moreover, there are different kinds of externalism: at the very least, vehicle externalism and content externalism. So, it is perhaps a good idea trying to deepen the kind of externalism involved here, assessing whether the relevant kind of externalism can actually vindicate the claim according to which the explanatory gap is removed.

Let us suppose, to begin with, that the sensorimotor approach is committed to externalism about intentional content. The idea of enactivists seems to be that it is impossible to disentangle purely intentional aspects from purely phenomenal aspects (see e.g. Noë, 2004: Chap. 3). Or, perhaps, they think that there are no phenomenal properties that are not intentional either (but see below). Yet there are many authors (for example, Chalmers, 1996) who take phenomenal properties as not supervenient on intentional properties. If these authors were right, externalism about intentional content would not imply externalism about phenomenal content. Therefore, two possibilities are open to the sensorimotor theorist: either she endorses the intentionalist theory of mental states, according to which phenomenal properties can be reduced to intentional properties-there are no phe-

\footnotetext{
${ }^{4}$ Since there are many versions of externalism, this claim should be qualified (see infra). For the moment, by "externalism of phenomenal facts" we intend the thesis that phenomenal consciousness does not supervene on the brain alone.
}

nomenal properties over and above the intentional ones-or she can try to show that even phenomenal properties (qua distinct from the intentional ones) are not supervenient on cerebral properties.

The second strategy is not much promising, because, as we saw in the previous section, the standard strategy to vindicate content externalism consists in claiming that a distal causal factor is constitutive of content; however, if this could sound reasonable for intentional content (at least in the case of perception), it is not equally plausible for phenomenal content. In fact the very idea of distinguishing intentional aspects from phenomenal aspects gives a prima facie reason for thinking that the latter supervenes on purely internal factors. In any case, as we saw in the previous section, it is hard to find in the enactivist essays an argument for thinking otherwise.

As to the first strategy, it would arguably be more promising. However, there is no clear evidence that it is endorsed by sensorimotor theorists. After all, they present their view as also able to account for phenomenal consciousness, i.e., for the qualitative properties; this seems to suggest that they are disposed to acknowledging some kind of (non-reducible) difference between intentional aspects and phenomenal aspects. Moreover, if they were actually intentionalist (that is, if they held that phenomenal states can be reduced to externally individuated intentional states), then, in order to remove the explanatory gap, they could simply argue for a functionalist treatment of intentional content—of course the relevant kind of functionalism would be a wide one (cf. Wilson 2004) ${ }^{5}$.

In sum, the inference from externalism on intentional content to externalism of phenomenal content does not seem to be a much manageable strategy for the sensorimotor theorist. Even if enactivists are certainly committed to perceptual content externalism, there is no easy way from here to the removal of the explanatory gap. Let us take into consideration, then, vehicle externalism ${ }^{6}$.

Enactivists are clearly committed to vehicle externalism. This commitment is a consequence of the thesis that perception, far from being a linear bottom-up processing flow, is a collection of looping interactions involving neural circuitry (connecting "higher" to "lower" brain areas, and encompassing both cognitive and motor systems) and bodily actions (Hurley, 1998; cf. also Clark, 2009: Section 5). On this view, the physical vehicles of experiential content (so, the subpersonal processes and events) are extended dynamic loops ${ }^{7}$.

\footnotetext{
${ }^{5}$ Arguably, the insistence on the temporal dimension of experience and the related remark that taking experience as a sequence of "snapshots" is misleading make somewhat unpalatable the notion of content itself (but it is worth to point out that Noë, 2004 uses systematically the word “content”).

${ }^{6}$ Sometimes, sensorimotor theorists appear to be inclined to eliminativism. Cf. e.g., "There is no explanatory gap. We do not claim that it is possible to explain the physical basis of conscious experience by appeal to sensorimotor contingencies. How, one might ask, can sensorimotor contingencies explain phenomenal consciousness any better than other proposals that have been made? Rather, we argue, as should by now be clear, that the conception of phenomenal consciousness itself must be (and can be) rejected, and so there is no longer any puzzle about how to explain that” (O’Regan \& Noë, 2001: p 963). This oscillation (which can also be attributed to Dennett, 1991) can perhaps be explained by different interpretations of what one means by “experiential properties”. For instance, O’Regan \& Noë (2001: p. 960) hold that it is one thing to say that (perceptual) experience has a qualitative character, and quite another to say that there are some properties, the qualia of experiential states: qualia do not exist, whereas the so-called experiential states are actually ways of acting; experience is something we do and its qualitative features are aspects of this activity.
} 
Now, we think that this view is a bit vague, and that much research has still to be done in order to get a reasonably clear picture of how all this works (the traditional picture is perhaps wrong, but is much clearer). However, independently of what are the perspectives for the dynamical systems theory in cognitive science-the kind of models clearly implied by the extended loops view-one can suppose that extra-cranial factors are part and parcel of the processes underlying our perceptual experience.

Suppose, then, to grant all that. We could still ask whether this is enough to claim that the explanatory gap is removed. It seems to us that it is sufficient to escape the causal-constitutive fallacy, but not sufficient to remove, or deflate, the explanatory gap, since the brain is still a relevant component of the process. Patently, nothing in the extended dynamic loops picture allows to rule out the possibility that the minimal machinery underlying conscious experience is limited to the processes running into the brain.

\section{Sensorimotor Subjectivity}

Evan Thompson $(2005,2007)$ has recently put forward an original proposal which, although belonging to the same sensorimotor/enactive framework illustrated in the first paragraph, differs from it in some aspects. We will offer two criticisms of this proposal. They are strictly related, but whereas the former has to do with Thompson's hypothesis of a "pre-reflective bodily self-consciousness" (PBSC from now on), the latter deals with the primacy of the first person perspective (with respect to the third person), that the author seems to advocate in cognitive science (in Dennett's 1991 terms, we could talk of the primacy of phenomenology over heterophenomenology).

Thompson introduces the concept of PBSC as the solution to the "body-body problem", that is, the problem concerning the relation between the lived-body, the body as a subject of action and cognition, and the living-body, the body as a biological object. According to Thompson (2005: p. 409), if the problem of consciousness is put this way, the gap is no longer "between two radically different ontologies (physical and mental), but between two types within one typology of embodiment (subjectively lived body and living body)". Filling this gap seems to be much less hard. The notion of PBSC would allow the re-connection of the living body-the body as an observable object-with the lived body, i.e., the body as a subject, the transcendental condition ${ }^{8}$ for the living body itself.

There is indeed a clear difference between feeling our own arm while we are touching it with our hand and feeling our hand touching the arm. But we maintain that the main shortcoming of the concept of PBSC is exactly the same as that of the Kantian transcendental; that is, its formal character, or, in other words, its empirical emptiness. Supposing that the bodybody problem is a more tractable problem than the mind-body (mental-physical) one, it is not at all clear why postulating a PBSC (as Kant postulated an "I THINK that must be able to accompany every representation of mine”) should be a more

\footnotetext{
${ }^{7}$ According to Clark (2009), this argument from "dynamic entanglement” is the best argument for the externalism of phenomenal content. More on this in next section.

${ }^{8}$ Here Thompson (2005: p. 410) refers explicitly to the notion of "transcendental" in Kant, remarking that within the phenomenological tradition (in particular Merleau-Ponty and Husserl) the transcendental is identified with the function of the lived-body.
}

satisfactory solution. In fact, the problem seems in this way to be displaced rather than solved: from the subjectivity of the lived body to the PBSC ${ }^{9}$.

Establishing an analogy between the concept of PBSC and the concept of "biological selfhood", typical even of the most elementary forms of life-the prokaryotes-cannot be of any help either. This kind of selfhood, according to Maturana and Varela (1987)'s autopoiesis thesis (to whom Thompson appeals here), rests upon the distinctive autonomy of living systems, which consists in their being constituted by their own biological processes. These processes neatly separate the organism, producing boundaries/membranes, from the rest of the world. In multicellular organisms endowed with a nervous system (metazoans, that is, animals), the biological identity is determined by the way the brain cyclically couples movement and sensory information (by means of the above-mentioned "sensorimotor loops"), disclosing a point of view or a perspective on the world (2005: pp. 418-419). It is worth to point out that, according to the autopoiesis thesis, biological processes are cognitive processes, so much so that there's no need for an organism to be endowed with a nervous system in order to have knowledge (of course, here we are dealing again with a sort of knowing-how, rather than a knowing-that). But, if so, what are the origin and the function of this alleged PBSC? Are humans the only organisms endowed with it? Maybe primates? Animals in general? It is not at all clear.

Note that, so far, Maturana, Varela and Thompson's picture does not seem to diverge from the account offered by Dennett, an author still sympathetic to the computational paradigm. Indeed, talking about the first forms of replicators, which are similar to the first forms of living being, prokaryotes, Dennett claims:

"When an entity arrives on the scene capable of behavior that staves off, however primitively, its own dissolution and decomposition, it brings with it into the world its 'good'. That is to say, it creates a point of view from which the world's events can be roughly partitioned into the favourable, the unfavourable and the neutral. And its own innate proclivities to seek the first, shun the second and ignore the third, contribute essentially to the definition of the three classes. As the creature thus comes to have interests, the world and its events begin creating reasons for it-whether or not the creature can fully recognize them" (1991: p. 189).

But, once such a minimal biological selfhood is accepted, either the concept of PBSC turns out to be redundant, given that it doesn't add much to it, or, as we said, it is empirically vacuous, since it merely postulates a formal (conscious) unity simply superimposed on the biological identity. In both cases PBSC lacks any explicative power.

This corroborates our judgment that the capacity of sensorimotor theories to deal with the explanatory gap depends essentially on the endorsement of vehicle externalism, notwithstanding Thompson's insistence on his being able to explain the subjective side of experience by means of PBSC. One could say, indeed, that Thompson's argument entailing the presence of a subjective pole in front of an objective one (holding intentional

\footnotetext{
${ }^{9}$ Our remark is sympathetic with Dennett's (2007: p. 267) criticism of the same concept: "What is this remarkable implicit awareness or pre-reflective self-consciousness? Thompson doesn't say [...] I want to substitute 'Newtonian' questions: what does implicit awareness $d o$ ? [...] What kind of things can a subject do that she wouldn't be able to do if it weren't for the gift of pre-reflective self-consciousness?”
} 
and phenomenal aspects together, as hypothesized by the intentionalist account) is logically valid: if we represent consciousness as a two-place relation, once we identify an object, we have to admit a subject who is conscious of that object. He writes (2005: p. 420) that, when I touch a bottle "The intentional object of my tactual experience is the bottle, but at the same time I live through my grasping feeling in a non-intentional (non-object-directed) manner. To experience the feeling as mine I do not have to identify it as mine. Instead the feeling comes with an intrinsic 'mineness' or first-personal giveness that constitutes its subjectivity”. But, again, Thompson is not clear about which requirements are needed in order to have a PBSC. Is it enough to have a body without a nervous system? It doesn't seem so, because, as we saw, the role of the nervous system is to couple sensory surfaces and effectors already to the aim of preserving the biological selfhood. But, then, Thompson cannot use twice this description, once for the biological selfhood, and the second time for the PBSC. Is the involvement of the environment also necessary for the PBSC? But, if so, Thompson should give us a deeper account of his standpoint with regards to active externalism (see above).

We agree, on the other hand, with Thompson (2005: p. 424) in rejecting O'Regan and Noë's claim that everything outside of the focus of attention is not conscious (2001: p. 964). This in fact presupposes an "all-or-nothing” view of consciousness that, on the other hand, O'Regan and Noë themselves criticize in other passages. Take Block's famous example ${ }^{10}$, in which a background noise never heard up to that point by a man absorbed in a conversation is suddenly noticed, and recognized as having been heard even before it was noticed. Thompson rejects both Block's (1997: p. 386) interpretation, according to which the man was phenomenally conscious of the noise, without having access consciousness of it, and O'Regan and Noë's interpretation, according to which, since you can't have phenomenal consciousness without access consciousness, the man couldn't be conscious of the noise before noticing it. Thompson thinks that the man had some conscious access to the noise because consciousness (PBSC) comes in a series of degrees, that is, has a graded structure, which should not be collapsed on the extreme pole of attention, the point in which the man comes up and exclaims "there it is. I hear it!".

This leads us to the second of the objections we mentioned at the beginning of this section, concerning the quest for the first person authority. The debate on this issue has been particularly lively over the last thirty years, but we will restrict ourselves to discussing a single point: if Thompson, like Dennett, is willing to propose a distinction of degree among levels of consciousness, shouldn't he also embrace the method of heterophenomenology, worked out by Dennett precisely in order to limit the first person pretention of knowing better than anyone else what is happening inside him? Accepting that consciousness is articulated in a variety of levels entails, indeed, that sometimes the subject of experience will not be able to verbally report what he is experiencing, as also Thompson is ready to admit. There are cases, as in Block's example, in which the subject succeeds in verbalizing it. But let's suppose that the man in his example never succeeds in realizing - nor, then, in reportingthat there was a background noise, up to that moment implicitly

\footnotetext{
${ }^{10}$ This is an example that has many variations in the XX century history of philosophy, from Husserl and Merleau-Ponty until the debate between Dretske (1994) and Dennett (1994) about non epistemic seeing, or "about differences that make no difference".
}

experienced. Who could demonstrate that the noise has ever been experienced by the subject? Dennett's heterophenomenological method (1991: Ch. 4, 2007) consists of giving the greatest credit to subjects' accounts about their experience, but only up to the point where experimental data contradict them.

In our variation on Block's example, while the subject could deny having ever heard any noise, a series of elements gathered by the scientist could contradict him, for example the fact that the man moved away from the source of noise, in order to better continue his conversation, or the fact that, asked about the intimacy of the place in which he was chatting, he answered that the place was not at all intimate. No view from within could ever settle this matter.

Therefore, Thompson's appeal to the phenomenological tradition, though relevant to the goal of improving first person descriptions ${ }^{11}$ (which represent the explanandum of a science of consciousness), shouldn't make us forget the various Dennettian insights about the fact that "there are circumstances in which people are just wrong about what they are doing and how they are doing it. It is not that they lie in the experimental situation, but that they confabulate, they fill in the gaps, guess, speculate, mistake theorizing with observing” (1991: p. 109). As we hinted above, this doesn't imply a complete acceptance of Dennett's skepticism about phenomenal aspects of consciousness, which, on the contrary, seemed to us originnally dealt with by the authors belonging to the sensorimotor front. But the fact that Thompson renamed "pre-reflective bodily self-consciousness" what Dennett and the majority of the neuroscientists usually call "consciousness" or "subjectivity” doesn't really seem to add anything to the explanation of the phenomenon.

\section{Conclusion}

The sensorimotor paradigm provides some insights concerning the problem of consciousness. In particular, the notion of virtual presence and the related features of grabbiness and bodiliness offer some suggestions aiming to restate the problem of phenomenal consciousness so that it can be scientifically addressed. However, the claim that the explanatory gap is closed is strongly overstated, for at least two reasons. First, the enactivist treatment of the issues of virtual presence and TVSS does not seem to force us to get rid of the brain as a constituent of the supervenience basis of perceptual experience. At most, it shows that the supervenience basis also includes some external factors. Second, even if one endorses the kind of externalism involved in the so-called extended mind framework (Clark and Chalmers's vehicle externalism), still there is no clear evidence that the brain plays a constitutive role, rather than a merely causal/instrumental one, in the production of phenomenal consciousness.

Similar considerations can be done for Thompson's notion of PBSC. We showed indeed that the alleged role played by PBSC in a theory of subjectivity can, in part, be filled by the same notion of biological selfhood that Thompson borrows from autopoiesis theory. However, the enactivist approach has undoubtedly the merit of highlighting the role of bodily compe-

\footnotetext{
${ }^{11}$ Thompson, Noë and Pessoa (1999) already stressed this point about the phenomenological shortcomings of Dennett's (1991) description of the filling-in phenomenon in the blind spot. Dennett admitted his descriptive mistakes, but pointed out that nothing in these authors' remarks refutes the heterophenomenological method (2005: p. 163).
} 
tence in perception, providing some insights to the study of conscious experience.

\section{REFERENCES}

Bach-y-Rita, P. (1972). Brain mechanisms in sensory substitution. New York: Academic Press.

Ballard D. H. (1996). On the function of visual representation. In K. Akins (Ed.), Perception (pp. 111-131). Oxford: Oxford University Press.

Ballard, D. H. (1991). Animate vision. Artificial Intelligence, 48, 57-86. http://dx.doi.org/10.1016/0004-3702(91)90080-4

Block, N. (1997). On a confusion about a function of consciousness. In N. Block, O. Flanagan, \& G. Güzeldere (Eds.), The nature of consciousness (pp. 375-416). Cambridge, MA: MIT Press.

Botvinick, M., \& Cohen, J. D. (1998). Rubber hand "feels" what eyes see. Nature, 391, 756. http://dx.doi.org/10.1038/35784

Brooks, R. (1991). Intelligence without representation. Artificial Intelligence, 47, 139-159. http://dx.doi.org/10.1016/0004-3702(91)90053-M

Brooks, R. (1999). Cambrian intelligence. Cambridge, MA: MIT Press.

Chalmers, D. (1996). The conscious mind. Oxford: Oxford University Press.

Churchland, P. S., \& Ramachandran, V. S. (1996). Filling in: Why Dennett is wrong. In K. Akins (Ed.), Perception (pp. 132-157). Oxford: Oxford University Press.

Clark, A. (1997). Being there. Cambridge, MA: MIT Press.

Clark, A. (1999). Visual awareness and visuomotor action. Journal of Consciousness Studies, 6, 1-18.

Clark, A. (2008). Supersizing the mind: Embodiment, action, and cognitive extension. Oxford: Oxford University Press.

http://dx.doi.org/10.1093/acprof:0so/9780195333213.001.0001

Clark, A. (2009). Spreading the joy? Why the machinery of consciousness is (probably) still in the head. Mind, 118, 963-993. http://dx.doi.org/10.1093/mind/fzp110

Clark, A., \& Chalmers, D. (1998). The extended mind. Analysis, 58, 10-23. http://dx.doi.org/10.1093/analys/58.1.7

Dennett, D. C. (1969). Content and consciousness. London: Routledge \& Kegan Paul.

Dennett, D. C. (1991). Consciousness explained. Boston: Little Brown \& $\mathrm{C}$.

Dennett, D. C. (1994). Get real. Philosophical Topics, 22, 505-568. http://dx.doi.org/10.5840/philtopics1994221/212

Dennett, D. C. (2005). Sweet dreams: Philosophical obstacles to a science of consciousness. Cambridge, MA: MIT Press.

Dennett, D. C. (2007). Heterophenomenology reconsidered. Phenomenology and the Cognitive Sciences, 6, 247-270. http://dx.doi.org/10.1007/s11097-006-9044-9

Dretske, F. (1994). Differences that make no difference. Philosophical Topics, 22, 41-58. http://dx.doi.org/10.5840/philtopics1994221/216

Fodor, J. A. (1980). Methodological Solipsism considered as a research strategy in cognitive science. Behavioral and Brain Sciences, 3, 63109. http://dx.doi.org/10.1017/S0140525X00001771

Gibson, J. J. (1979). The ecological approach to visual perception. Boston: Houghton Mifflin.

Hurley, S. (1998). Consciousness in action. Cambridge: Harvard University Press.

Hurley, S. (2001). Perception and action: Alternative views. Synthese,
129, 3-40. http://dx.doi.org/10.1023/A:1012643006930

Hurley, S., \& Noë, A. (2003). Neural plasticity and consciousness. Biology and Philosophy, 18, 131-168. http://dx.doi.org/10.1023/A:1023308401356

Kanizsa, G. (1979). Organization in vision. New York: Praeger.

Kelso, S. A. (1995). Dynamic patterns. Cambridge, MA: MIT Press.

Levine (1983). Materialism and qualia: The explanatory gap. Pacific Philosophical Quarterly, 64, 354-361.

Lycan, W. (1987). Consciousness. Cambridge, MA: MIT Press.

Maturana, H., \& Varela, F. (1987). The tree of knowledge. Boston: Shambhala.

McGinn, C. (1989). Mental content. Oxford: Blackwell.

Metzinger, T. (2003). Being no one. The self-model theory of subjectivity. Cambridge, MA: MIT Press.

Myin, E., \& O’Regan, K. (2008). Situated perception and sensation in vision and other modalities. In P. Robbins, \& M. Aydede (Eds.), Cambridge Handbook of Situated Cognition (pp. 185-200). Cambridge: Cambridge University Press.

Noë, A. (2004). Action in Perception, Cambridge, MA: MIT Press.

Noë, A. (2006). Experience without the head. In T. S. Gendler, \& J. Hawthorne (Eds.), Perceptual experience (pp. 411-434). Oxford: Oxford University Press. http://dx.doi.org/10.1093/acprof:oso/9780199289769.003.0012

Noë, A., \& Thompson, E. (2004). Are there neural correlates of consciousness? Journal of Consciousness Studies, 11, 3-28.

O’Regan, K., Myin, E., \& Noë, A. (2005). Sensory consciousness explained (better) in terms of "corporality" and "alerting capacity". Phenomenology and the Cognitive Sciences, 4, 369-387. http://dx.doi.org/10.1007/s11097-005-9000-0

O'Regan, K., \& Noë, A. (2001). A sensorimotor account of vision and visual consciousness. Behavioral and Brain Sciences, 24, 939-1011. http://dx.doi.org/10.1017/S0140525X01000115

Ramachandran, V., \& Blakeslee, S. (1998). Phantoms in the brain. New York: William Morrow.

Thelen, E., \& Smith, L. (1994). A dynamical systems approach to the development of cognition and action. Cambridge, MA: MIT Press.

Thompson, E. (2005). Sensorimotor subjectivity and the enactive approach to experience. Phenomenology and the Cognitive Sciences, 4, 407-427. http://dx.doi.org/10.1007/s11097-005-9003-x

Thompson, E. (2007). Mind in life. Cambridge, MA: Harvard University Press.

Thompson, E., \& Varela, F. (2001). Radical embodiment: Neural dynamics and consciousness. Trends in Cognitive Sciences, 5, 418-425. http://dx.doi.org/10.1016/S1364-6613(00)01750-2

Thompson, E., Noë, A., \& Pessoa, L. (1999). Perceptual completion. In J. Petitot et al. (Eds.), Naturalizing phenomenology. Stanford, CA: Stanford University Press.

Thompson, E., Palacios, A., \& Varela, F. (1992). Ways of coloring: Comparative color vision as a case study for cognitive science. Behavioral and Brain Sciences, 15, 1-74. http://dx.doi.org/10.1017/S0140525X00067248

Varela, F., Thompson, E., \& Rosch, E. (1991). The embodied mind: Cognitive science and human experience. Cambridge, MA: MIT Press.

Wilson, R. A. (2004). Boundaries of the mind. The individual in the fragile sciences: Cognition. Cambridge, MA: Cambridge University Press. 